\title{
Welcome to Journal of Modern Materials
}

\section{A New Open Access Journal for International Exchange of Scientific Research}

Received: 5 April 2016 / Published: 12 June 2016

Keywords: Editorial, Journal of Modern Materials, Editorial Welcome

Materials science research has been the most important field of research for a long time. Only materials scientists can provide the building blocks, modelling, necessary tools, processes, and components that are needed for an ever-growing scientific society. Materials science research is a multidisciplinary field, where physicists, chemists, engineers, biologists, and others meet to explore the properties, structure, and applications from a single atom or molecule to a complex structure such as nanostructured materials. Dealing with problems in the materials field requires the development of sophisticated new equipment and procedures. Recently, advanced materials such as functional materials, photovoltaic materials, conductive polymers, composite materials, nanostructured materials, etc. have attracted remarkable attention.

All over the world, more and more researchers are involving in various aspects of materials science research starting from simulation to synthesis, characterization and proceeding into application in engineering and medical fields. As the materials research field is ever broadening, increasingly specialized journals have appeared. However, in order to enable successful interactions between different fields, the need for journals that cover all aspects of materials science research is crucial. Moreover, with the rapidly growing research community, there is a necessity to provide free and easy access to high-quality research and innovations. This can be achieved by introducing open access journals in all research fields. Open access journals do have several advantages, such as free access to everyone, the inclusion of colour figures at no extra cost, rapid publication, global access, and hence, the long-term impact can be expected. So, a new open access journal, "Journal of Modern Materials [JMM]" published by AIJR should be of interest to researchers working in the field of materials synthesis, characterization, and application.

Therefore, I am inviting you to submit your original research articles to the Journal of Modern Materials and hope that with time Journal of Modern Materials will become a premier publication for materials research worldwide. This goal can be achieved with the cooperation of our editorial team, reviewers, and authors to publish such a high-quality original research that attracts attention. We warmly welcome authors to submit their research papers in the inaugural issue of this journal that is truly international in the scope which aims to publish manuscripts from all over the world.

I hope that you will enjoy reading the first issue of the Journal of Modern Materials. Your valuable feedback will be appreciated for improving the quality of the publication.

June 2016

Dr. Adam A. Bahishti

Editor,

Journal of Modern Materials

\section{How to Cite this Article:}

A. Bahishti, "Welcome to Journal of Modern Materials", J. Mod. Mater., vol. 1, no. 1, p. 1, Jun. 2016. doi: 10.21467/jmm.1.1.1 